\title{
Meticillin-heteroresistant Staphylococcus pasteuri from an apheresis platelet product
}

About 1 in 1000-2000 apheresis platelet units is contaminated by bacteria. The platelet products are stored in oxygenpermeable bags, at $20-24{ }^{\circ} \mathrm{C}$, for up to 5 days before being transfused, to preserve cell survival and function. Unfortunately, this may also permit growth of bacteria and transmission of these to recipients. Coagulase-negative staphylococci, Staphylococcus aureus, $\alpha$-haemolytic streptococci, diphtheroids, Propionibacterium acnes, Acinetobacter species, Escherichia coli, Serratia species, Klebsiella species, Enterobacter species, Providencia rettgeri and Yersinia enterocolitica are known to contaminate platelet units (Riedel et al., 2006; Savini et al., 2008). Though contaminating staphylococci generally exert $\beta$-lactam susceptibility (as they are mostly donors' skin contaminants, not nosocomial isolates), meticillin resistance should always be excluded to avoid $\beta$-lactam failure when treating transfusion-related bacteraemias.

A Staphylococcus pasteuri strain, designated Staphylococcus pasteuri A, was isolated as a contaminant from an apheresis platelet unit from an adult first-time donor. Identification was obtained by rRNA sequencing. Given that the screened unit had not yet been transfused, an aliquot from the apheresis bag was newly examined to exclude the possibility that contamination had occurred while inoculating the BacT/Alert bottles (bioMerieux BacT/Alert is the instrument which is used in our department to screen platelet products for bacterial contamination) (Riedel et al., 2006); interestingly, the same strain grew again. Antimicrobial susceptibilities were determined by bioMérieux Vitek2, and meticillin susceptibility was confirmed by a standard agar disc test (NCCLS, 2003). A $20 \mathrm{~mm}$ inhibition zone diameter was documented around the Oxoid meticillin disc, but two single colonies

(Staphylococcus pasteuri B and C) were observed within the inhibition area after $48 \mathrm{~h}$ incubation. These were both subcultured and showed the same phenotypic features as Staphylococcus pasteuri A (typical yellowish staphylococcal colonies), but exerted full meticillin resistance; an MIC $\geqslant 0.5 \mathrm{mg} \mathrm{l}^{-1}$ was documented by Vitek2, whereas an $8 \mathrm{~mm}$ diameter meticillin inhibition zone was obtained by carrying out a disc test (NCCLS, 2003). Meticillin resistance thus appeared to be heterogeneously expressed within the population studied, and the isolate was finally considered $\beta$-lactamresistant. The documented susceptibility profile was as follows: resistant to penicillin ( $\mathrm{MIC} \geqslant 0.5 \mathrm{mg} \mathrm{l}^{-1}$ ); heteroresistant to meticillin/oxacillin (MIC $\geqslant 0.5 \mathrm{mg} \mathrm{l}^{-1}$ ); susceptible to erythromycin $\left(\mathrm{MIC} \leqslant 0.25 \mathrm{mg} \mathrm{l}^{-1}\right.$ ), clindamycin (MIC $\leqslant 0.5 \mathrm{mg} \mathrm{l}^{-1}$ ), ciprofloxacin (MIC $\leqslant 0.5 \mathrm{mg} \mathrm{l}^{-1}$ ), cotrimoxazole (MIC $\leqslant 10 \mathrm{mg} \mathrm{l}^{-1}$ ), teicoplanin (MIC $1 \mathrm{mg} \mathrm{l}^{-1}$ ) and vancomycin (MIC $1 \mathrm{mg} \mathrm{l}^{-1}$ ).

Heteroresistance means the existence of a mixed population of drug-sensitive and drug-resistant organisms in a clinical isolate; in a most stringent sense, it is an exclusively phenotypic manifestation within a genotypically homogeneous strain. Heteroresistance may be undetected if only automated susceptibility tests are performed or only standard agar methods are carried out. This may lead to antimicrobial failure due to in vivo overgrowth of mutant drug-resistant clones. Meticillin and vancomycin heteroresistance has been found in Staphylococcus aureus, Staphylococcus epidermidis, Staphylococcus simulans, Staphylococcus haemolyticus, Staphylococcus capitis, Staphylococcus auricularis and Staphylococcus warneri. Gardnerella vaginalis metronidazole heteroresistance, Acinetobacter baumannii carbapenem heteroresistance and Cryptococcus neoformans fluconazole heteroresistance have also been reported in the literature. This phenomenon has also been described in Enterococcus faecium, Streptococcus pneumoniae and Mycobacterium tuberculosis, but never in Staphylococcus pasteuri. The frequency of heteroresistance is known to be about one subclone in every $10^{5}-10^{6}$ colonies, though this varies amongst different species. Heterogeneous resistance may represent a first step towards resistance, as it permits organisms to grow in the presence of an antibiotic, before the acquisition of resistance by the greater part of the microbial population (Falagas et al., 2008; Pournaras et al., 2005).

Heteroresistance is known to be difficult to detect, and there are few clinical data concerning this topic. Also,

heteroresistance may not be evident before $48 \mathrm{~h}$ incubation even if agar methods are used. Hence we would suggest that laboratories carefully screen bacterial organisms for heterogeneous resistance to antimicrobials in daily clinical practice. Though clinical antimicrobial failures due to undetected heteroresistance have been reported, the therapeutic implications of this phenomenon have to be clarified and further clinical and experimental data are required (Falagas et al., 2008).

Staphylococcus pasteuri is a coagulasenegative staphylococcus which contributes naturally to Italian sausage fermentation, and development of their typical colour and aroma (Iacumin et al., 2006). Also, Staphylococcus pasteuri is one of the few micro-organisms which have been cultured from the stratosphere (Wainwright et al., 2006). Concerning Staphylococcus pasteuri involvement in human disease, the organism has only recently been found to cause a proven bacteraemic episode in a leukaemia patient (Savini et al., 2009), whereas the clinical significance of the isolates recovered by Chesneau et al. (1993) remained doubtful. The occurrence of platelet unit contamination by this species, however, may make it gain importance as an agent 
of platelet-derived bacteraemias in the coming years. Also, widespread use of molecular methods for bacterial

identification based on rRNA sequencing will probably lead to an increase in the number of Staphylococcus pasteuri isolates recovered from blood products and clinical specimens, given that automated methods which are commonly used in routine practice may fail to recognize this species on the basis of the biochemical profile. There are many unanswered questions about Staphylococcus pasteuri. We presently know very little about its ecological niches and its ability to cause disease in animals and man and to acquire resistance determinants. Nevertheless, its capacity to exert $\beta$-lactam resistance is of concern. To the best of our knowledge, this is the second isolation of Staphylococcus pasteuri from a blood product (Savini et al., 2008). Our brief communication reports what we believe to be the first recovery of meticillin heteroresistance in the species discussed. This is thought to be due to the heterogeneous expression of the mecA gene within the bacterial population, and this could lead to in vivo selection of fully $\beta$-lactam-resistant mutant clones if a transfusion-related bacteraemia occurred.
Vincenzo Savini, ${ }^{1}$ Azaira Bianco, ${ }^{1}$ Chiara Catavitello, ${ }^{1}$ Andrea Balbinot, ${ }^{1}$ Arianna Pompilio, ${ }^{2}$ Raffaele Piccolomini, ${ }^{2}$ Giovanni Di Bonaventura, ${ }^{2}$ Antonio lacone ${ }^{3}$ and Domenico D'Antonio ${ }^{1}$

${ }^{1}$ Clinical Microbiology and Virology Unit, Department of Transfusion Medicine, 'Spirito Santo' Hospital, Pescara, Italy

${ }^{2}$ Clinical Microbiology, Aging Research Center (CeSI), and Department of Biomedical Sciences, 'Gabriele d'Annunzio' University of Chieti-Pescara, Italy

${ }^{3}$ Department of Transfusion Medicine, 'Spirito Santo' Hospital, Pescara, Italy

Correspondence: Vincenzo Savini

(vincsavi@yahoo.it)

Chesneau, O., Morvan, A., Grimont, F., Labischinski, H. \& El Solh, N. (1993).

Staphylococcus pasteuri sp. nov., isolated from human, animal, and food specimens. Int J Syst Bacteriol 43, 237-244.

Falagas, M. E., Makris, G. C., Dimopoulos, G. \& Matthaiou, D. K. (2008). Heteroresistance: a concern of increasing clinical significance? Clin Microbiol Infect 14, 101-104. lacumin, L., Comi, G., Cantoni, C. \& Cocolin, L. (2006). Ecology and dynamics of coagulasenegative cocci isolated from naturally fermented Italian sausages. Syst Appl Microbiol 29, 480-486.

NCCLS (2003). Performance Standards for Antimicrobial Disk Susceptibility Tests, 8th edn. Approved Standard M2-A8. Wayne, PA: National Committee for Clinical Laboratory Standards.

Pournaras, S., Ikonomidis, A.,

Markogiannakis, A., Maniatis, A. N. \& Tsakris, A. (2005). Heteroresistance to carbapenems in Acinetobacter baumannii. J Antimicrob Chemother 55, 1055-1056.

Riedel, S., Siwek, G., Beekmann, S. E., Richter, S. S., Raife, T. \& Doern, G. V. (2006). Comparison of the BACTEC 9240 and BacT/ Alert blood culture system for detection of bacterial contamination in platelet concentrates. J Clin Microbiol 44, 2262-2264.

Savini, V., Catavitello, C., Pompetti, F., Passeri, C., Di Zacomo, S., Esattore, F., lacone, A. \&

D'Antonio, D. (2008). Contamination of a donated platelet unit by Staphylococcus pasteuri. J Infect 57, 494-496.

Savini, V., Catavitello, C., Carlino, D., Pompilio, A., Balbinot, A., Piccolomini, R., Di Bonaventura, G. \& D'Antonio, D. (2009). Staphylococcus pasteuri bacteraemia in a leukemic patient. J Clin Pathol (in press).

Wainwright, M., Alharbi, S. \& Wickramasinghe, N. C. (2006). How do microorganisms reach the stratosphere? Int J Astrobiol 5, 13-15. 